DOI: http://doi.org/10.52716/jprs.v11i4.564

\title{
Improving the Performance of Split Air Conditioner by Adding Nano Silver to the Compressor Oil
}

\author{
Abdulqadir Hameed Rasheed ${ }^{1, *}$, Adnan M. Hussein ${ }^{2}$ \\ ${ }^{1}$ The state company for gas filling and services. \\ ${ }^{2}$ Northern Technical University, College of health and medical technology \\ 1,* Corresponding Author E-mail: qaderrasheed@gmail.com \\ 22dradnan_hwi@ntu.edu.iq
}

Received 11/4/2021, Accepted 26/5/2021, Published 20/12/2021

This work is licensed under a Creative Commons Attribution 4.0 International License.

\begin{abstract}
$\underline{\text { Abstract }}$
A high demand on the energy and power has brought scholars more attention to investigate new ways to reduce the energy consumption. The aim of this research is reducing the power consumption required for air conditioning to cool the room by adding nanomaterials to the compressor oil at a mass concentration of $0.15 \%$. Two models of chambers with dimensions $(2 \times 2 \times 2)$ meters were fabricated to represent the test section and connected to a 1 ton compressor, one operating with natural oil as a standard basis for comparison, while the other one with Nano-oil prepared in the laboratory. The mass concentrations of $0.15 \%$ of silver nanoparticles (Ag) were prepared, and the stability of the nanoparticles was tested by direct observation for different time periods. The results indicated that the performance of the air conditioner was better with the addition of nanoparticles to the compressor oil compared to the pure oil in the compressor. Reducing energy consumption in the cooling system by (19\%). Also, it was found that there is an increase in the cooling speed of the nanotechnology system by (6\%)as well as in the efficiency of the Nano-oil system compared to the regular system by (13\%). This study showed that the thermophysical properties of the cooling oil increased by increasing the weight ratios of solid nanoparticles by $(12 \%)$.
\end{abstract}

Keywords: air conditioning, nanoparticles, silver, Nano-oil, thermophysical properties. 


\section{تحسين أداء مكيف الهواء عن طريق إضافة الفضة النانوية إلى زيت الضاغط}

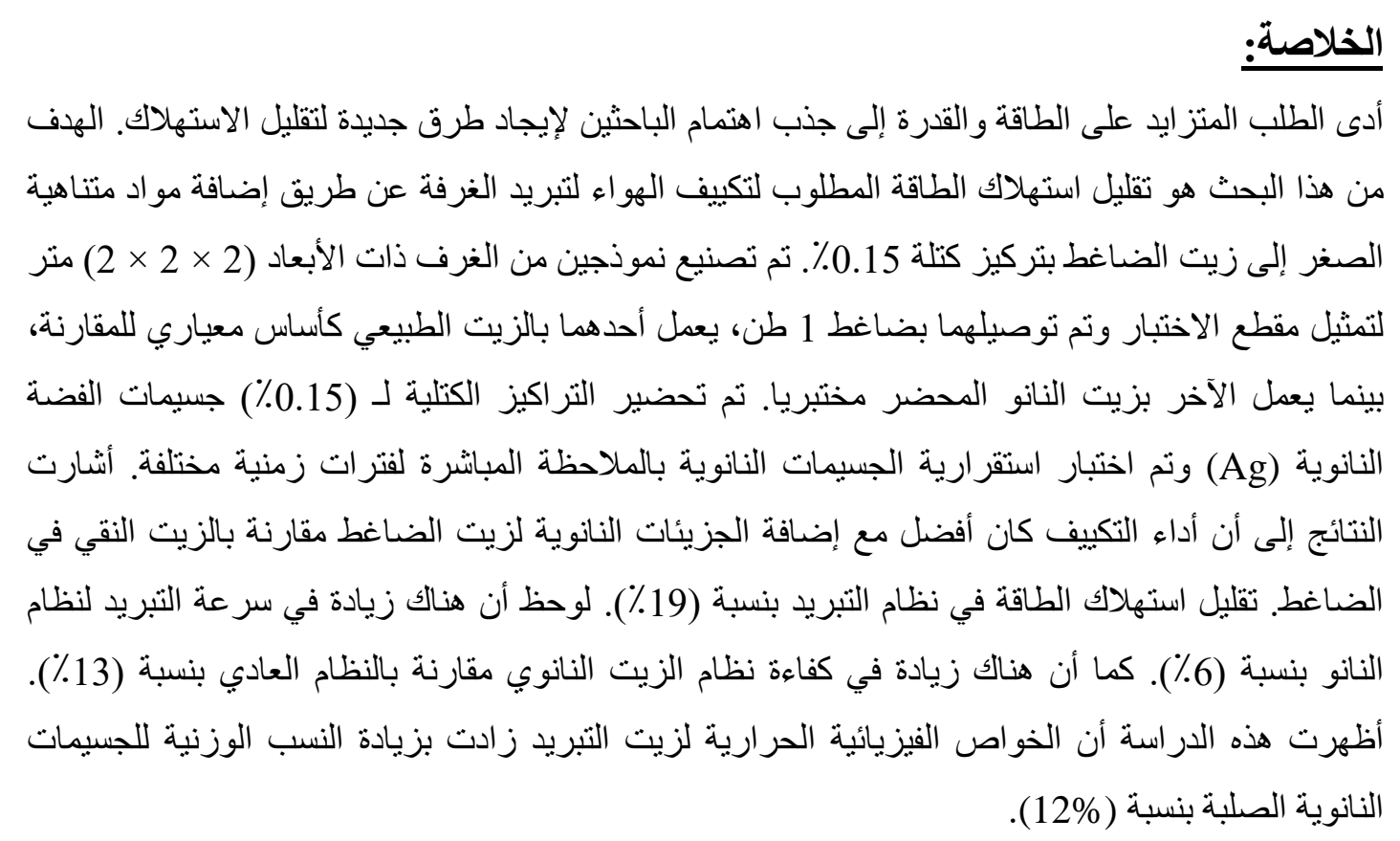

\section{Introduction}

Air conditioning is the process of treating the surrounding atmosphere by controlling the level of temperature, humidity, and air movement inside the place to be adapted, and to obtain an appropriate atmosphere in which the person is feeling comfortable and the appropriate temperature. The field of refrigeration and air conditioning is one of the fields of continuous prosperity in the world, especially in developing countries where no industrial or scientific field that does not depend in one respect on the science of refrigeration and air conditioning.

Since ancient times, Egyptians used pottery vessels to cool drinking water. Then, a group of Eskimo men discovered the body of an extinct animal long ago buried in the ice in good condition. Then using ice spread to preserve food in its areas. For thousands of years, humans have used natural ice to do the cooling process. [1]

American Jacob Perkins obtained a patent in 1834 for a closed-cycle compressor refrigeration machine that used ethyl alcohol as a cooling medium. Then, in 1844, the physicist John Gorrie developed a compressive cooling machine 
that uses air as a cooling medium, to produce ice and cold air. In 1850 AD, a French man named Edmund Carre designed small cooling machines from them that paved the way for the manufacture of artificial ice, which affected the natural ice trade. [2]

At the beginning of the twentieth century and with the advent of electricity, the refrigeration and air-conditioning industry made a great progress that everyone feels at our time, so that almost no home at this time is devoid of an electric refrigerator. In the near future, we may not find a house without an air conditioner. This is a short historical overview of refrigeration, and from it we see that the year has surpassed nature in finding cooling at anytime and anywhere when a person asks. [3]

The cooling process can be accomplished by utilizing any physical process, accompanied by an absorption of heat such as: phase transitions of the material (accompanied by heat absorption such as melting or evaporation), throttling (where gases pass through constrictions or valves), expansion of gases and cyclones in tubes, and demagnetization of the body Steel. [4]

Nanotechnology is defined as metal and non-metal solid particles with a diameter of less than 100 nanometers and new behaviors and characteristics. Because these particles (smaller than the lengths of properties associated with certain phenomena) exhibit different chemical and physical concepts. The electronic structure of materials, mechanical properties, conduction, interaction and fusion change as the particle size changes to the nanoscale value. The size of the material might be changed its engineering properties, thus investigators explained its excellent technical effects in various fields by reducing the time of nanoscale delivery to the human circulation, producing light and strong materials, and improving the size of magnetic absorption strips then computer keyboards will be faster[5, 6].

The most significant shape of carbon nanotube is one of spherical shape that substance (C60) photons category, while they are slightly different from the composition as they are multi-crust with an empty center unlike nanoparticles, and may be not any surface gaps between Multi-cover nanotubes [7]. 
Some researchers studied the performance of air conditioner and refrigerator for improving the energy consumption by $(26 \%)$ with mixing ratio $(0.1 \%)$ of $\mathrm{TiO} 2$ solid nanoparticles using in the hydrofluorocarbon (HFC134) system and Polyester oil (POE oil).

Number of studies was performed alumina nanoparticles that showed the different nanoparticles characteristics have slightly influence on the refrigerator efficiency. It was observed that the solid nanoparticles might be used for refrigerators domestic applications to save energy consumption. It was noted that there are two ways for the impact of nanoparticles on performance of air conditioner and refrigerator: one of them is improving friction factor due to remain nanoparticles inside compressor. While the second is improving the heat transfer due to pass the nanoparticles through the heat exchanger [7, 8].

The alumina nanoparticles viscosity dispersed in liquids like as water and ethylene Glycol. Results illustrated that viscosity is increasing due to increase in nanoparticles fraction as compared to base fluid. [9]

In the last decade, number of works were conducted on a refrigeration system by using different lubricants, refrigerants. It was indicated that the nanoparticles are significant consumption of power economically and enhance of performance coefficient (COP) of the system. [10]

Nowadays, nanotechnology has become significant and rousing fields in physics, chemistry, engineering and biology. In the near future, the scientific revolutions have adopted the nanotechnology to great hope for changing technology direction for industrials' applications. During the years between 1996 and 1998, the nanotechnology has become impact interest for many researchers.

The American Global Technology Assessment Center (WTEC) performed an estimation of nanotechnology field investigating with importance of technical innovation. [11]

The recommendations of these investigating are the impact points of nanotechnology applications in military, medical, electronic, informatics, computer, petrochemical, biological and other fields. Therefore, investigators in the fields of 
nanotechnology must communicate with other fields, in order to draw a plan of nanotechnology background with active participation in this exciting field. [12]

In addition, the relevant administrators and the authors of this study should be familiar with a general summary of these areas. The nanotechnology concept has based on the assumption that the size particles is less than $100 \mathrm{~nm}$ give the material that included in its composition new properties and behaviors.

The reason of this assumption is the particles should be smaller than the lengths of properties led to some behavior to introduce new chemical and physical concepts, leading to new behavior dependent on size of particle. It can be noted that the melting point, conductivity, reactivity, electronic composition and mechanical properties of all change material when falling the particle size below a critical size value.

As the matter approaches volume the dimensions of atomic, more it obeys the quantum mechanics laws rather than the traditional physics laws. The behavior dependence of the material on its diameter enables us to control the physical properties, and regarded to the conclusion of investigators that the behavior of nanoparticles is more technical implications that include varied and wide fields that include the tough and light particles production, shortening the delivery time of nanoparticles to the system of human circulatory, improving the absorption volume of magnetic tapes and computer preparation hot keys.

In general, nanotechnology is one that deals with multiple combinations of materials with dimensions of the order of nanometer. [13]

Most of the electrical energy consumed is through refrigeration and airconditioning equipment. Here, the importance of finding solutions to reduce electrical energy consumption in the world and especially in Iraq, due to the hot and dry weather that characterizes Iraq's climate in summer.

The literature reviewing is including the nanofluid applications by using alumina, titania, and copper with cooling systems. The gap is application of silver nanofluid with air conditioner especially with gas R410 which there was no researcher focused it. The novelty of this research is the utilizing of silver nanofluid in air conditioner with gas R410 which friendship with environment. 
The research aims to use silver nanoparticles suspended in pure oil in air conditioner that contains gas R410. Two test rigs were used to conduct experiments for the sake of comparison. One of them contains air conditioner device with pure oil, while the other has the same specifications with 0.001 silver nanoparticles suspended in oil. The improvement of the amount of electrical energy consumption and the efficiency of the system has been studied.

\section{2- Materials and Methodology}

\section{1. the main assumptions were made based on the following:}

- Assuming steady- state heat transfer and fluid flow.

- The flow is three-dimensional and single phase in the system.

- Assuming constant properties of nano-oil and laminar fluid flow.

\subsection{Experimental test rig}

In order to measure the Nano-oil and oil density, the container volume shown in Figure (1) is $25 \mathrm{ml}$ and weight of $21.39 \mathrm{~g}$ then adding oil to be $43.99 \mathrm{~g}$ as following [14]:

$\rho_{\text {oil }}=\frac{m}{v}$

$M=43.99-21.39=22.6 g$

$V=25 m l$

$\rho_{\text {oil }}=\frac{22.6}{25}=0.904 \frac{\mathrm{g}}{\mathrm{cm}^{3}}$

$\rho_{0.0015}=\frac{m}{v}$

$M=276.66-91.62=185.04 g$

$V=200 \mathrm{ml}$

$\rho_{0.001}=\frac{185.04}{200}=0.925 \frac{\mathrm{g}}{\mathrm{cm}^{3}}$

$\rho_{0.001}=(1-\emptyset) \rho_{f}+\emptyset \rho_{n}$ 


$$
\rho_{0.001}=(1-0.0015) 0.904+0.001 * 10.49=0.913 \frac{g}{\mathrm{~cm}^{3}}
$$
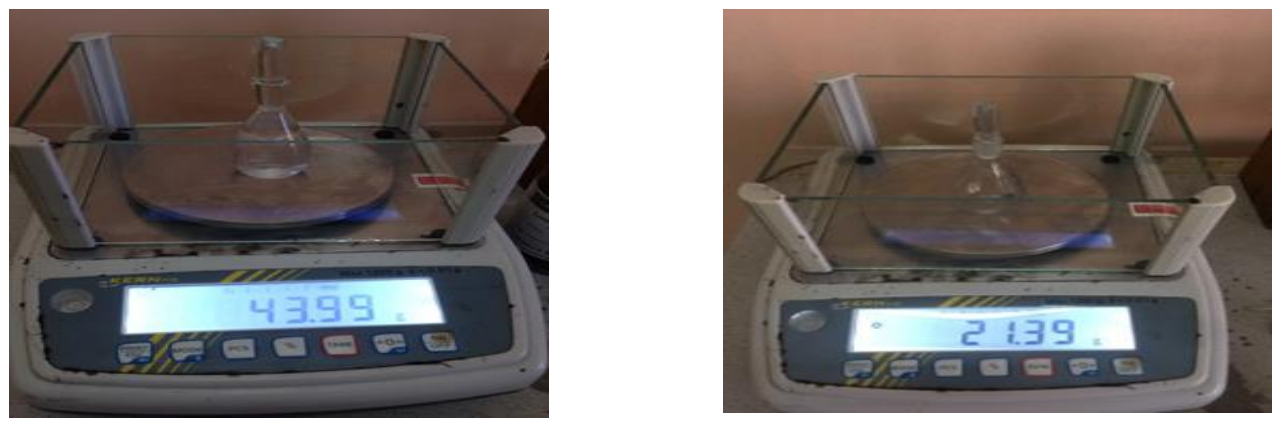

(a) weighing process for pure oil.
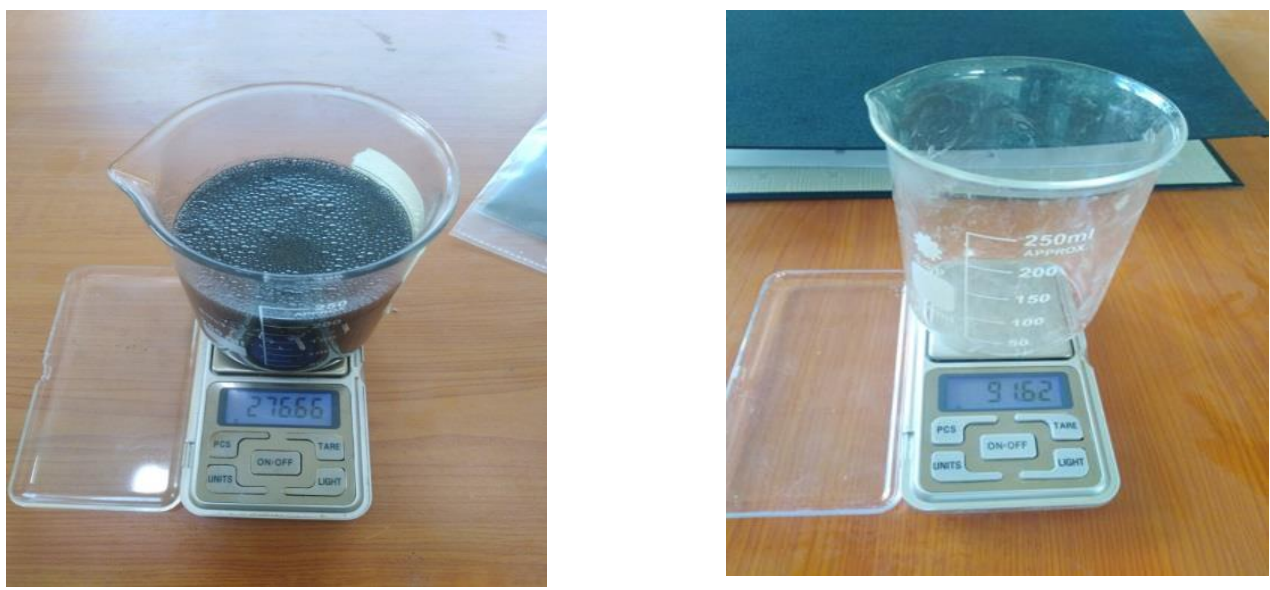

(b) weighing process for Nano-oil.

Fig. (1) Weighing process for pure oil and Nano-oil.

To evaluate thermal conductivity of oil and Nano-oil, the device shown in Figure (2) has been used. It was observed $12.56 \mathrm{~W} / \mathrm{m} . \mathrm{K}$ thermal conductivity of silver Nano-oil measured by device whereas the thermal conductivity of pure silver was $429 \mathrm{~W} / \mathrm{m} . \mathrm{k}$ as summarized in Table (1). [14]

$k_{n}=k_{f}\left(\frac{k_{s}+2 k_{f}-2 \emptyset\left(k_{f}-k_{s}\right)}{k_{s}+2 k_{f}+\emptyset\left(k_{f}-k_{s}\right)}\right)$ 


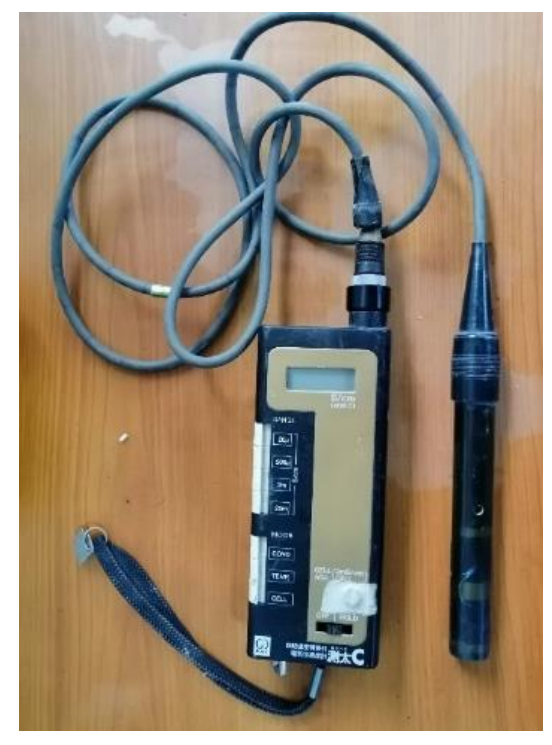

Fig. (2) Thermal conductivity device.

Table (1) thermophysical properties of solid nanoparticles [4]

\begin{tabular}{lccc}
\hline Material & $\begin{array}{c}\text { Density } \\
\left(\mathbf{k g} / \mathbf{~ m}^{3}\right)\end{array}$ & $\begin{array}{c}\text { Thermal Conductivity } \\
(\mathbf{W} / \mathbf{m} . \mathbf{k})\end{array}$ & $\begin{array}{c}\text { Specific heat } \\
(\mathbf{J} / \mathbf{k g . k})\end{array}$ \\
\hline Silver & 10490 & 429 & 710 \\
Copper & 8954 & 380 & 390 \\
Aluminium & 2700 & 237 & 910 \\
Diamond & 3510 & 3300 & 425 \\
Carbon nanotubes & 2250 & 3000 & 410 \\
Silicon & 2330 & 148 & 710 \\
Alumina $\left(\mathrm{Al}_{2} \mathrm{O}_{3}\right)$ & 3880 & 36 & 773 \\
Silica $(\mathrm{SiO} 2)$ & 2220 & 1.4 & 745 \\
Titanium dioxide $\left(\mathrm{TiO}_{2}\right)$ & 4175 & 8.4 & 692 \\
Water & 998.9 & 0.613 & 4181 \\
Ethylene glycol & 1110 & 0.253 & 2200 \\
Engine oil & 890 & 0.145 & 1800 \\
\hline
\end{tabular}

The viscosity of liquids is a great property to describe the potential of liquids and applications. Digital Rotational Viscosity Meter shown in Figure (3) has been used to measure the liquid viscosity experimentally. [14].

$$
\mu_{n f}=\mu_{b f}(1+2.5 \emptyset)
$$




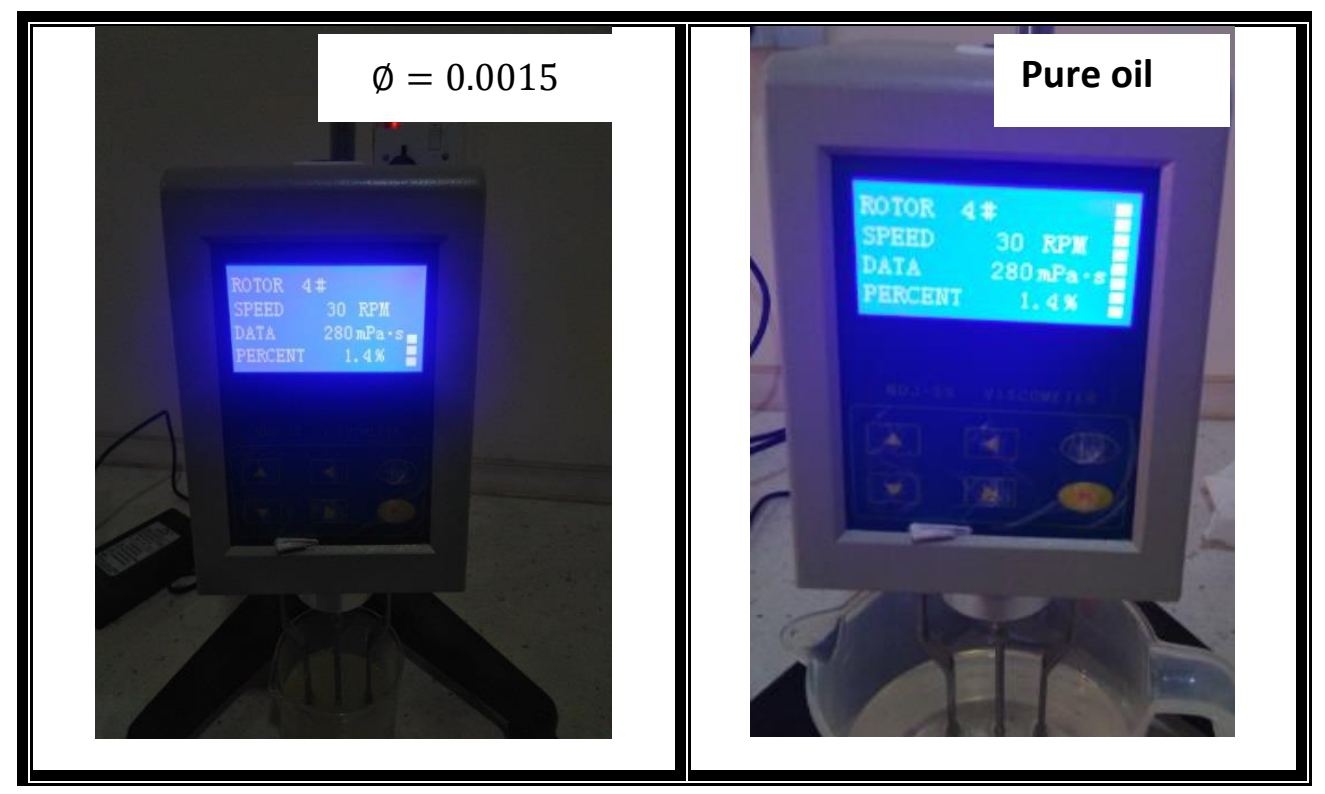

Fig. (3) viscosity measurement.

The efficiency of cooling systems and heat pumps are indicated by the Performance Coefficient (COP). COP is evaluated by the ratio between the energy use of the compressor and the useful amount of cooling in the evaporator or the useful heat rejected from the condenser (for the heat pump). A high COP value represents high efficiency.

C. O.P $=\frac{\mathrm{QE}}{\mathrm{W}}$

\subsection{Nano-Oil Preparation Methods}

The solid nanoparticles are purchasing and mixing with oil lubricant by two methods to be ready for experiments as following:

- Mechanical method:

An electric mixer has been used to disperse the solid nanoparticles in oil to evaluate quantities until it appears completely homogeneous. Along two hours the mechanical mixing has been applied to ensure homogenous oil lubricant.

- Ultrasonic device (Ultrasound):

The mixture of oil and nano solid particles in a mechanical way is placed in a number of flasks to obtain a homogeneous mixture (Hussein et al., 2013). Along one 
hour, the ultrasonically process has been applied. Then the observation process has been conducted by showing the samples after preparation and each hour till separation and sedimentation happen to ensure the stability of Nano-lubricant for utilizing in compressor.

$$
m_{p}=\frac{\emptyset \times \rho_{p} \times\left(\frac{m_{f}}{\rho_{f}}\right)}{(1-\emptyset)}
$$

\subsection{Calibration Process}

The calibration process is one of the most important processes took place in measurement laboratories. It is a measurement process that is carried out under a specific condition using measuring devices supported by international standards, and the process is carried out under conditions similar to the operational conditions of the device to be calibrated.

The purpose of the calibration process is to know the accuracy of measuring the devices used. The temperature measurement devices were calibrated with a mercury thermometer and the maximum readings deviation were approximately $2 \%$. The thermal conductivity device was calibrated by checking the thermal conductivity of water and the maximum deviation was $1 \%$.

\section{Results And Discussion}

Figure (4) shows the amount of electric current difference drawn through the system when using pure oil and nanoparticles with a mass concentration (0.0015). It turns out that the average electric current drawn through the system using pure oil was (4.8 A), while the average electric current drawn through the system using the Nanooil was (3.9A). This indicates that the use of Nano-oil was able to reduce the current consumption by (0.9 A). 


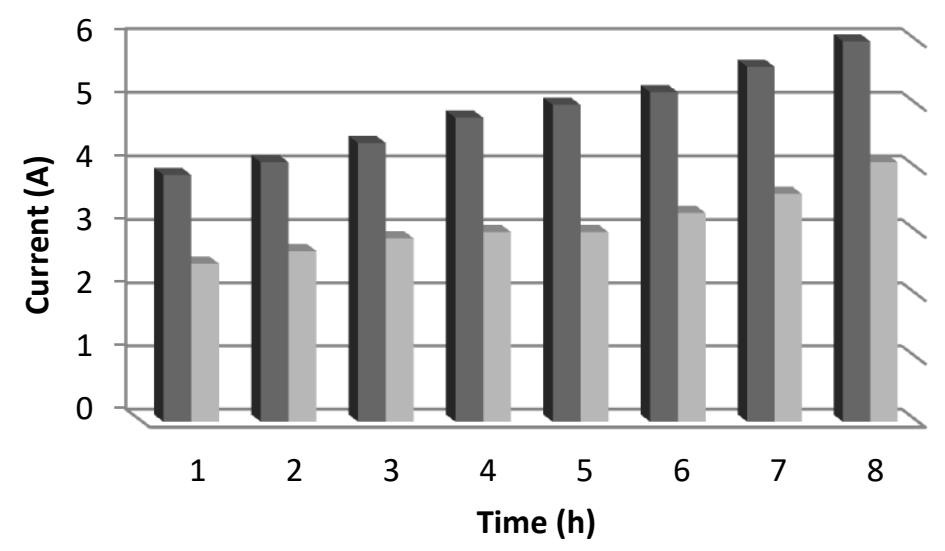

Fig. (4) Current consumption in split air conditioning with time.

It should be measuring the room temperature to assess the air conditioner equipment. The temperature change can be seen as in Figure (5) as the average room temperature, which was measured by thermocouples adjacent to the inner wall. It can be noted that the measured temperature is $19^{\circ} \mathrm{C}$ when using pure oil. The average temperature measured when using the Nano-oil was $16^{\circ} \mathrm{C}$. Note that the system using pure oil has started working at the temperature $40^{\circ} \mathrm{C}$, while the Nanooil system has started working at a temperature of $40^{\circ} \mathrm{C}$, this indicates the cooling speed of the Nano -oil system compared to the regular system.

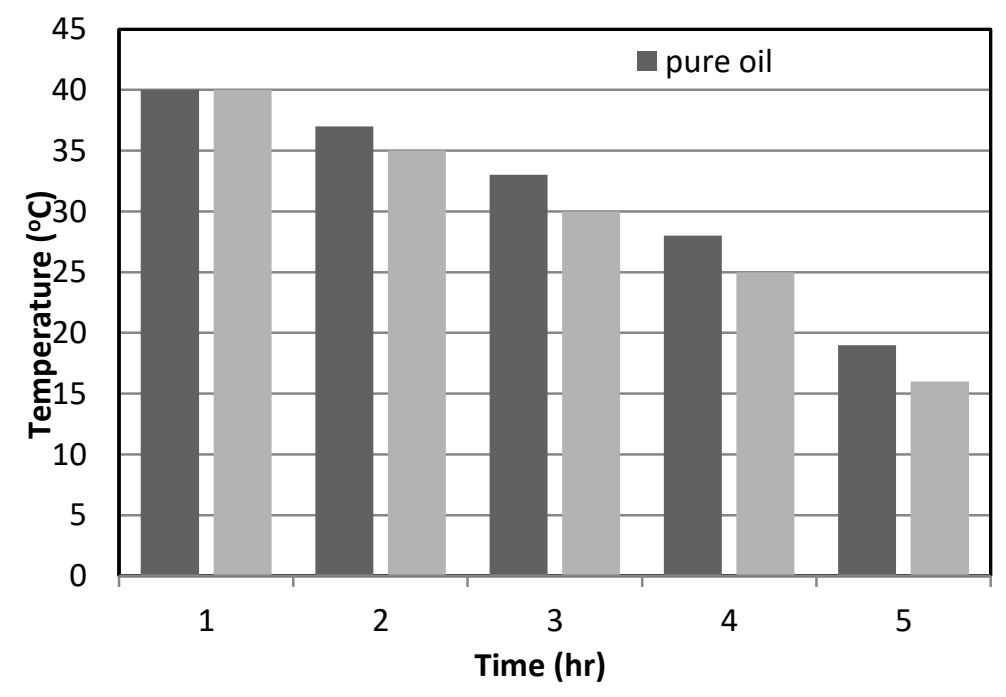

Fig. (5) Temperature change with time. 
Figure (6) shows the thermal performance parameter of a split cooling system using pure oil at one time and Nano-oil at other times. It can be seen that the performance parameter increases with increasing the weight ratio of the nanoparticles with the same system and the same ambient conditions. COP of split cooling system are 3.35 and 4.8 for using pure oil and Nano-oil respectively dur to increase in the weight ratio of the nanoparticles.

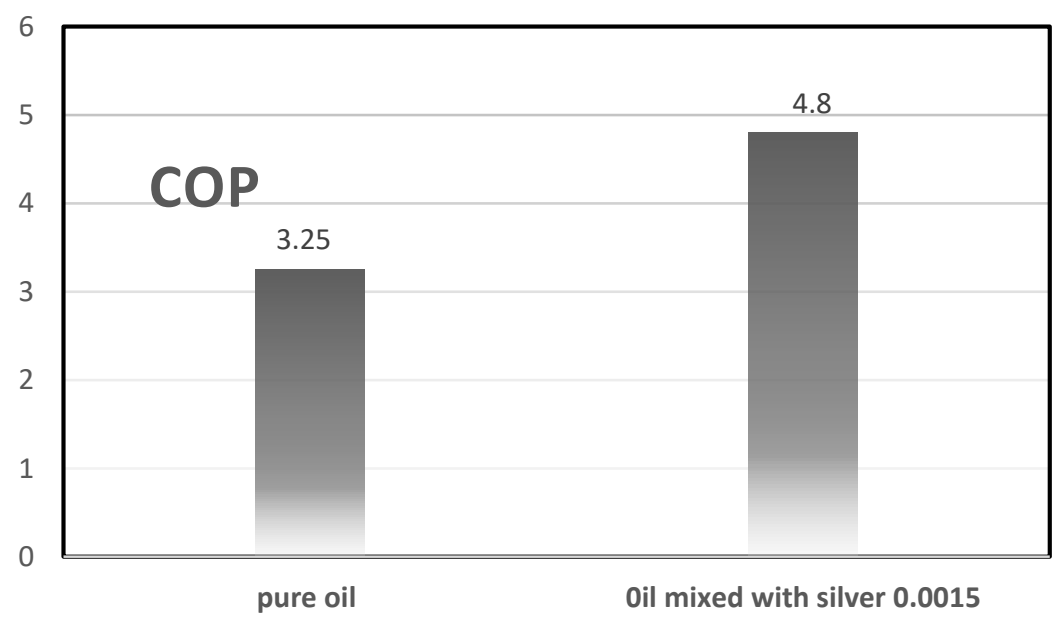

Fig. (6) Thermal performance comparison.

\section{Conclusions}

In order to reduce the power consumption required for air conditioning to cool the room, the solid nanoparticles have been added to the compressor oil at a mass concentration of $0.15 \%$. The following conclusions of this study summarized as:

- The electrical energy consumption through a split air conditioner has been reduced by $(19 \%)$.

- The speed of a split air conditioner using nanoscale silver has been increased as compared to a system operating with pure oil by $(6 \%)$.

- The thermal performance coefficient, which represents the efficiency of the Nano-oil has been increased as compared to the original system, by (13\%).

- The thermophysical properties is increased with increasing of the nanoparticles mass concentration by $(12 \%)$. 


\section{$\underline{\text { Symbols }}$}

$\rho_{p} \quad$ Density of nano atoms $\left(\mathrm{kg} / \mathrm{m}^{3}\right)$

$\rho_{\mathrm{f}} \quad$ Pure oil density $\left(\mathrm{kg} / \mathrm{m}^{3}\right)$

m Weight $(\mathrm{kg})$

$\mathbf{m}_{\mathbf{p}} \quad$ The nanoparticles weight $(\mathrm{kg})$

$\mathbf{m}_{\mathbf{f}} \quad$ Weight of oil $(\mathrm{kg})$

V Nanoparticles size $\left(\mathrm{m}^{3}\right)$

K Thermal conductivity (W/m.K)

$\mathbf{k}_{\mathbf{n}} \quad$ Thermal conductivity of nano-oil (W/m.K)

$\mathbf{k}_{\mathbf{f}} \quad$ Pure oil thermal conductivity $(\mathrm{W} / \mathrm{m} . \mathrm{K})$

$\mu_{\text {nf }} \quad$ The viscosity of the nano-oil (Pa.s)

$\mu_{\mathbf{b f}} \quad$ The viscosity of the pure oil (Pa.s)

$\phi \quad$ mass friction (\%) 


\section{$\underline{\text { References }}$}

1. Chand, R. , Rana , G. and Hussein, A.K. Effect of suspended particles on the onset of thermal convection in a nanofluid layer for more realistic boundary conditions, International Journal of Fluid Mechanics Research ,Vol. 42, No. 5, 2015, pp : 375-390.

2. Hamadalla M.W., Jumaah O.M., Shaalan Z.A., Hussein A.M., Performance Enhancement of Air Conditioning (Split Unit) Using CuO/Oil NanoLubricant. Materials Science Forum Submitted: 2020-06-19.

3. Hussein A.M., Kadirgama K., Noor M.M., Nanoparticles suspended in ethylene glycol thermal properties and applications: An overview, Renewable and Sustainable Energy Reviews 69 (2017) 1324-1330.

4. Hussein A.M., Sharma K.V., Bakar R.A., Kadirgama K., Heat transfer enhancement using nanofluids in an automotive cooling system, Int. Comm. in Heat and Mass Transfer 53 (2014) 195-202.

5. Azeez K., Hameed A.F., Hussein A.M., Nanofluid heat transfer augmentation in a double pipe heat exchanger. AIP Conference Proceedings, Vol. 2213, Issue 1, 2020.

6. Azeez K., Ibrahim Z.A., Hussein A.M., Thermal Conductivity and Viscosity Measurement of $\mathrm{ZnO}$ Nanoparticles Dispersing in Various Base Fluids. J.of Adv. Res. in Fluid Mech. Ther. Sci. 66(2), (2020) 1-10.

7. Wongwises, W, An experimental study on the heat transfer performance and pressure drop of $\mathrm{TiO} 2-$ water nanofluids flowing under a turbulent flow regime. International Journal of Heat and Mass Transfer 2010;53(1-3):33444.

8. Trisaksri V, Wongwises S. Nucleate pool boiling heat transfer of TiO2R141b nanofluids. International Journal of Heat and Mass Transfer 2009;52(5-6):1582-8.

9. Ahmed, S. , Hussein, A.K. , Mohammed, H. and Sivasankaran, S. Boundary layer flow and heat transfer due to permeable stretching tube in the presence 
of heat source/sink utilizing nanofluids, Applied Mathematics and Computation, Vol. 238, 2014, pp : 149-162.

10. Godson L, Raja, Mohan LD, Wongwises S. Enhancement of heat transfer using nanofluids - an overview. Renewable and Sustainable Energy Reviews 2010;14(2):629-41.

11. Hussein A.M., Sharma K.V., Bakar R.A., Kadirgama K., the effect of cross sectional area of tube on friction factor and heat transfer nanofluid turbulent flow, Int. Comm. in Heat and Mass Transfer 47 (2013) 49-55.

12. Jiang W, Ding G, Peng H. Measurement and model on thermal conductivities of carbon nanotube nanorefrigerants. International Journal of Thermal Sciences 2009;48:1108-15.

13. Chand, R. , Rana , G. and Hussein, A.K. On the onset of thermal instability in a low Prandtl number nanofluid layer in a porous medium, Journal of Applied Fluid Mechanics , Vol. 8 , No.2 , 2015, pp : 265-272

14. A.M. Hussein, K.V. Sharma, R.A. Bakar, K. Kadirgama, The effect of nanofluid volume concentration on heat transfer and friction factor inside a horizontal tube, J. of Nanomaterials 2013 (2013) 1-9. 